九州大学学術情報リポジトリ

Kyushu University Institutional Repository

\title{
Visualized IEC : Interactive Evolutionary Computation with Multidimensional Data Visualization
}

Hayashida, Norimasa

Kyushu Institute of Design

Takagi, Hideyuki

Kyushu Institute of Design

http://hdl. handle. net/2324/1670071

出版情報: IEEE International Conference on Industrial Electronics, Control and Instrumentation. 3, pp. 2738-2743，2000-10-25. IEEE

バージョン :

権利関係 : 


\title{
Visualized IEC: Interactive Evolutionary Computation with Multidimensional Data Visualization
}

\author{
Norimasa Hayashida Hideyuki Takagi \\ Kyushu Institute of Design \\ Shiobaru, Minami-ku, Fukuoka, 815-8540 Japan \\ Tel\&Fax: +81-92-553-4555, takagi@kyushu-id.ac.jp, http://www.kyushu-id.ac.jp/ takagi
}

\begin{abstract}
We propose Visualized IEC as an interactive evolutionary computation (IEC) with visualizing individuals in a multidimensional searching space in a 2-D space. This visualization helps us envision the landscape of an $n$-D searching space, so that it is easier for us to join an EC search by indicating the possible global optimum estimated in the 2-D mapped space. We experimentally evaluate the effect of visualization using a benchmark function. We use self-organizing maps for the projection of individuals onto a 2-D space. The experimental result shows that the convergence speed of GA with human search on the visualized space is at least five times faster than a conventional GA.
\end{abstract}

\section{Introduction}

Interactive evolutionary computation (IEC) has been used for several application tasks, for example, hearing aid fitting, speech processing, and 3-D CG lighting $[9,10,11]$. In spite of the increasing interest, this technology still has a common problem with these applications: the fatigue of IEC users. To make this IEC technology practical, we must improve the user interface.

In a conventional IEC, the roles of EC and a human user are completely separated; the EC performs the searches while the human user evaluates the result. The user plays a passive role in the search which contributes to the slow convergence and human fatigue.

We propose a method that allows an IEC user to directly participate in EC searches to ease psychological and physical fatigue. The active user intervention leads to a faster convergence of the EC search, and the faster convergence results in less user fatigue. On-line knowledge embedding is one based on this idea. This method provides a mechanism to accept the searching idea, hints, or intentions of an IEC user during the IEC operation $[2,12]$. For example, when a user feels that a certain part image of a montage face is acceptable, we fix the partial face image in subsequent searches, which limits the searching space and, therefore, con- verges faster. The effect of this method was shown through subjective tests [12]. Directly editing the tree of genetic programming for CG is another method of active user intervention to EC search [14].

Our proposed Visualized IEC is another method based on the same idea of active user intervention. The Visualized IEC allows an IEC user to actively participate in EC searches by providing the user the distribution image of past individuals mapped from a $n$-D searching space to a 2-D space. We first propose the Visualized IEC and preliminarily evaluate its convergence performance using a benchmark function.

\section{Visualized IEC}

\subsection{Why Visualized IEC ?}

Visualized IEC is a method that combines the different capabilities of EC and humans to search for a global optimum. The EC directly and systematically searches the original $n$-D space using EC operators, which is much better than the human searching capability. However, humans have an excellent capacity to grasp an entire distribution of individuals in the 2-D space at a macroscopic level that cannot be interpreted by the EC. This is why the Visualized IEC combines the algorithmic EC search in an $n$-D space and the human global search in a mapped 2-D space.

Since a human IEC user and the EC cooperate with each other and optimize their own searching advantages in the Visualized IEC, we can expect a faster convergence. This searching cooperation feature is different from a conventional IEC or previously proposed visualization-based searches. The roles of the EC and the human are separated in conventional IEC; the EC performs the search and an IEC user evaluates the searched individuals, independently. For example, the visualization-based searches used in a 3-D CG design support system [7] and a violin sound estimator [6] request human users to both search for the global optimum on the mapped 2-D searching space without the help of optimization algorithms and evaluate the searched individuals. 


\subsection{Multidimensional Data Projection}

Since it is difficult for humans to grasp the geometric relationship among individuals that have multidimensional parameters, it is difficult to directly obtain information from an $n$-D searching space. However, it becomes easier for humans to grasp the approximate relationships among individuals by mapping the individuals from the $n$-D searching space to a $2-\mathrm{D}$ space. Although the 2-D space does not keep all the information about the original $n$-D space, humans can grasp the state of the whole of the searching space when the topological relationships among individuals in the 2-D space are about the same as those in the original $n-\mathrm{D}$ space, which helps humans to cooperate with the EC on search and indicate the direction to the global optimum. The indication of the search is based on the geometric relationships kept which shows that individuals with high fitness values tend to concentrate on the same points in the 2-D space (see Figure 1.)

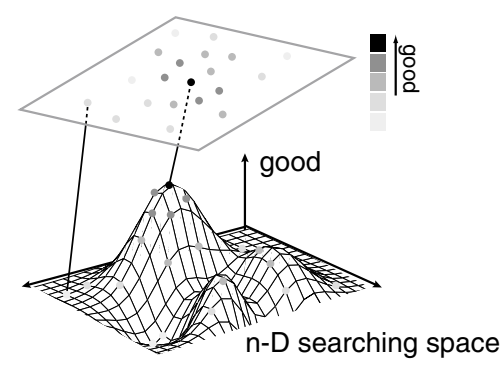

Figure 1: Projection image from an $n$-D space to a 2-D space while keeping the topological relationships among data samples.

There are several mapping methods for 2-D visualization, for example, the principle component analysis, Sammon's non-linear mapping [8], self-organizing maps [3], Visor [4], TOPAS [5], and the method using Genetic Programming [13]. Any visualization methods are usable in the Visualized IEC, but some methods are more suitable for the Visualized IEC than others. We need to evaluate the differences in their performance as our next research topic.

\subsection{Construction of a Visualized IEC system}

In the IEC, EC optimizes the parameters of an application task, and the task system outputs sound, speech, graphics, or other outputs which deals with the human senses. The human IEC user observes the output, evaluates them based on his or her sense of value, and returns his or her subjective fitness value to the EC (see Figure 2 upper.)
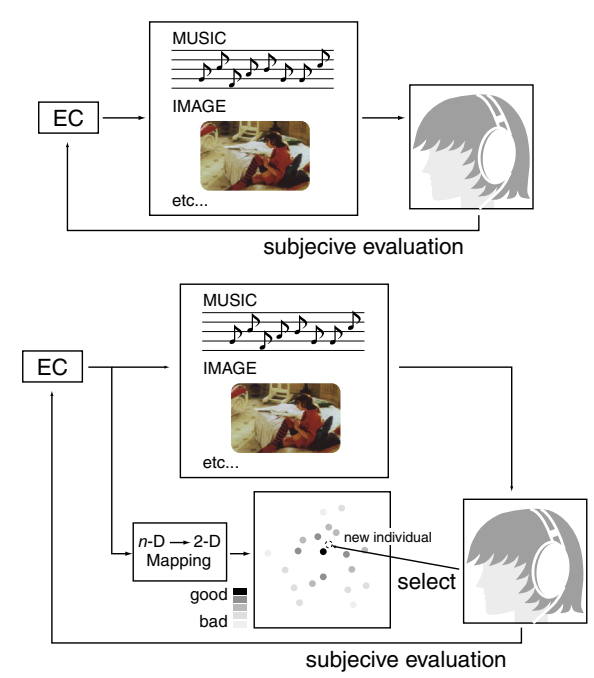

Figure 2: Diagrams of IEC (upper) and Visualized IEC (lower).

Visualized IEC systems display not only sound or graphics to the user, but also the distribution image of past EC searched individuals by mapping them from an $n$-D searching space to a $2-\mathrm{D}$ space (see Figure 2 lower.) Fitness values of individuals in the 2-D space are displayed with different colors, the depth of color, size, or numerical characters to view, for example, the landscape shape of a searching space. An IEC user selects points in the $2-\mathrm{D}$ space that look to have high fitness values from the fitness value distribution point of view, and the individual with the lowest fitness value in the EC population is replaced with the selected best point. Since neighboring individuals in an $n$-D space becomes neighbors in a 2-D space too, the topological relation of the $n$-D space is kept in the mapped 2-D space and the additional selected individual in the 2-D space can be expected to be located near the global optimum in the $n$-D space. The EC mates and creates offspring using the parent population that includes the newly added individual.

Since the possibility that an excellent individual is added to the EC population in each generation by the IEC user is high, the acceleration of EC convergence is expected.

\section{Evaluation of Convergence}

\subsection{Experimental System}

We evaluate how human intervention accelerates the convergence of the EC search. The final evaluation should be conducted using the Visualized IEC and subjective tests. Since the IEC deals with subjective fitness values that depend on the application task and the 


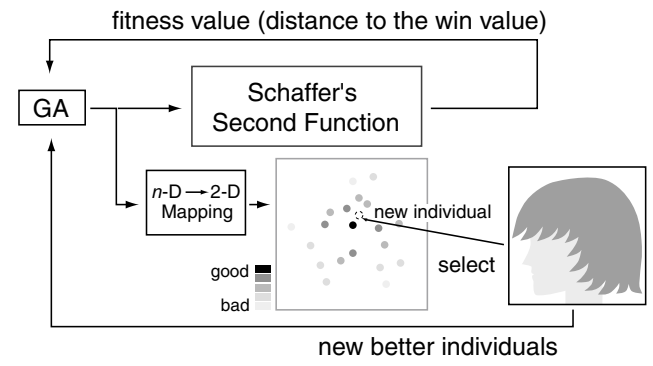

Figure 3: Experimental system of the Visualized GA. GA determines the coordinate of the minimum value of the Schaffer's second function, and the difference of the function output and the minimum value is fed-back into the GA as a fitness value. The human operator visually selects a possible global optimum in the mapped 2-D space and sends it to the GA as a new possible parent. Self-organizing map is used to map individuals from an $n$-D space to a 2 -D space.

subject's perceived value of the task, we preliminarily evaluate the effect of the human intervention without subjective evaluation to the given tasks in this section.

We do not compare the convergence performance of the Visualized IEC and IEC but that of the Visualized $\mathrm{EC}$ and EC in reality; since we use genetic algorithms (GA) as one of the EC technologies in this section, the actual comparison is the Visualized GA versus a normal GA. This experimental evaluation needs no human interactive evaluation of the given task but only the human selection of better individuals in the 2-D space.

Figure 3 shows an experimental system. Since this system has a fitness function, the role of the human user is only to select better candidates in the 2-D visualized space, while a Visualized IEC user plays both roles of a fitness function and a selector of the better candidates (compare Figures 2 and 3.)

Two modified Schaffer's second functions illustrated in Figure 4 in 3-D and 5-D are used as the experimental tasks of the Visualized GA and GA. The task is to determine the coordinate that results in the minimum value of the Schaffer's function, i.e. 0. Distance, $\mid$ function( GA individual ) - $0 \mid$, is fed-back into the $\mathrm{GA}$ as a fitness value.

Figure 5 is the interface design of the mapped 2D space used in our experiment. The fitness values of individuals, including those from past generations, are displayed with different depths of color. A human user finds an area where individuals with higher fitness values gather, selects a maximum of three individuals that seem to have the highest fitness values, and replaces one of the 20 individuals with the selected one.

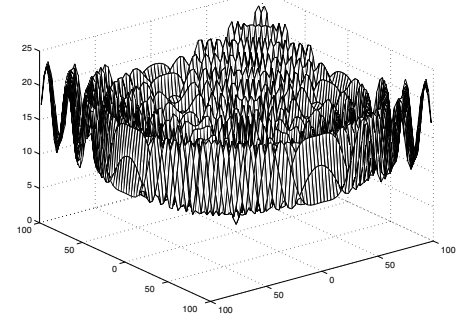

Figure 4: Modified Schaffer's second function given by $\left(\sum_{i=1}^{n} x_{i}^{2}\right)^{\frac{1}{4}} *\left\{\sin ^{2}\left(50 *\left(\sum_{i=1}^{m} x_{i}^{2}\right)^{\frac{1}{10}}+1\right\}\right.$, where $-100 \leq x_{i} \leq 100$ and $n=3$ and 5 .

Besides the classification by the five levels of depth of blue color, our experimental system can display the exact fitness value of the selected point so it is easier for users to compare individuals. The best individual in each generation is displayed with an orange color, and if the fitness value of a new individual created by the user is better than these best individuals, the new one is displayed in pink.



Figure 5: The example image of the mapped 2-D space of 40 individuals ( $=20$ individuals $\times 2$ generations $)$ in the second generation and some individuals created by a user. Original interface distinguishes individuals with color.

We adopt self-organizing maps (SOM) to map individuals from an $n$-D space to a 2 -D space. Figure 5 is the example of mapped distribution of individuals. The population size is 20 . As the amount of data increases, we re-train the SOM in every generation, so that the number of displayed individuals in the 2-D space increases 20 by 20 in each generation. The possible mapped points in the 2 -D space are $200 \times 200$.

The experimental conditions of GA and SOM are showed in Table 1. The evaluating subjects in our experiment are five graduate and undergraduate students. 
Table 1: Experimental conditions of GA and SOM.

(a) GA parameters

\begin{tabular}{|c|c|}
\hline population size & 20 \\
crossover rate & 0.9 \\
mutation rate & $1 / 80$ \\
\# of generations & 10 \\
bit length & 16 \\
\hline
\end{tabular}

(b) SOM parameters

\begin{tabular}{|c|c|}
\hline \# of learning & 1,000 \\
neighborhood function & step function \\
shape of neighborhood & hexagon \\
neighborhood radius & 5 \\
learning rate factor & 0.4 \\
\# of units & $200 \times 200$ \\
\hline
\end{tabular}

\subsection{Experimental Results}

Figure 6 shows the experimental results for the tasks whose complexity is different, where the dimensional number, $n$, is 3 and 5 . These graphs clearly show that the visualized GA converges much faster than a normal GA; the convergence speed of the Visualized GA with a population size of 20 is similar to that of normal GA with a population size of 100 and 1,000. This means we can expect that the Visualized GA to converge five time faster or more than the normal GA.

\subsection{Discussion}

Although the convergence characteristics of our proposed method depends on the subjects, the average convergence characteristics of the five subjects was much faster than that of normal GA. Due to human fatigue, the number of EC generations and the number of individuals displayed to an IEC user was usually limited to 20 generations and 20 individuals at most. Therefore, the experimental result that the proposed method with a few individuals converges faster than the normal GA with many individuals implies that the Visualized IEC is expected to be a powerful tool for difficult IEC tasks.

Generally, the higher the dimensional number of a searching space, the more slowly the GA converges. In our experimental evaluation, the convergence performances of some experimental subjects did not depend on the dimensional number, 3 and 5 . There was even a case that convergence in a 5 -D space was better than that in 3-D space. We can expect that the convergence of the Visualized IEC is tolerant about the complexity of tasks and that the Visualized IEC becomes much more powerful for complex tasks.

In early generations, when the individuals are sparsely displayed in the 2-D space, it is often difficult to observe the landscape of the searching space, which makes
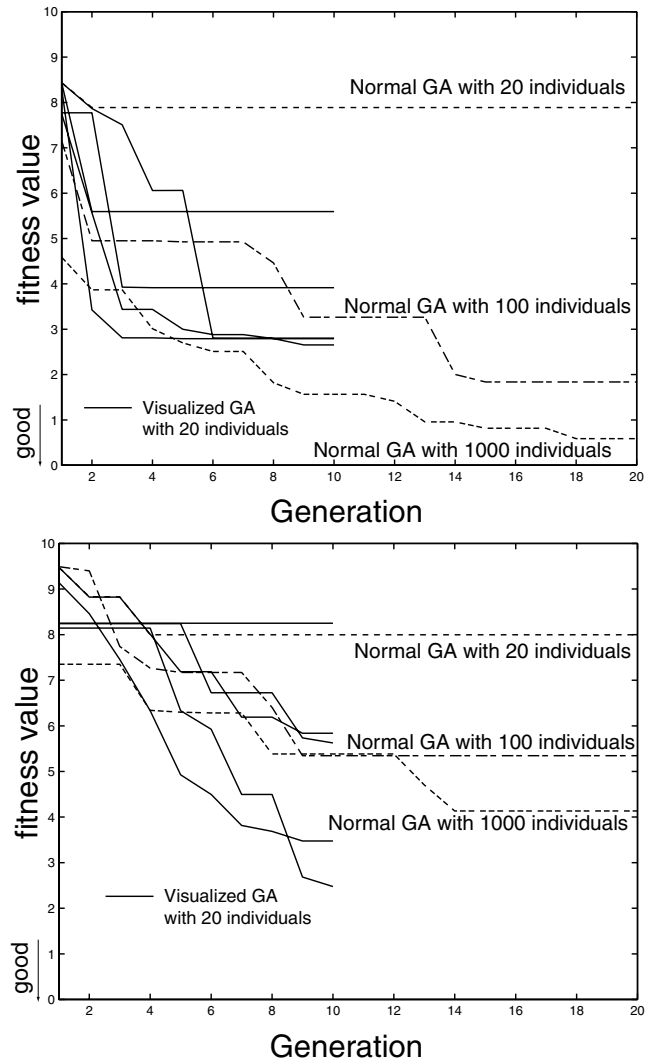

Figure 6: Convergence characteristics of normal GAs with population sizes of 20,100 , and 1,000 and the Visualized GA with a population size of 20 for a modified Schaffer's function of 3-D and 5-D, are shown in the upper and lower graphs, respectively. Solid lines represent the five experimental subjects for the Visualized GA.

it difficult for human subjects to estimate the location of the global optimum in the 2-D space. For such a case, they choose points near the best individuals in the 2-D space. As these points are expected to have higher fitness values as well as the best individuals, the sparse distribution in the early generation may not been a serious problem.

Even if IEC users select and create a worst individual, it mean that only one bad individual is added to many in a population, and it is weeded out by the natural selection in the next generation, which is not a serious problem.

In our experiment, we found that the training time of SOM to map 40,000 points $(=200 \times 200$, see Table 1 (b)) in every generation would be too long for a smooth human interaction with the computer. There are two solutions: reducing the number of SOM units 
and adopting a better mapping method. After the experiment described in this section, we confirmed that the SOM calculation time could be decreased within an allowable range without reducing its performance by reducing the number of SOM units. In our second experiment, we used the SOM as the first step. There are several projection methods as mentioned in section 2.2 that have different visualization and calculated time characteristics. We are going to compare these methods in our next step.

\section{Applications of Visualized IEC}

The experimental evaluation in the previous section has shown the effectiveness of visualization for GA search, and we can expect the Visualized IEC is a practical solution for several applications. In this section, we show two examples of Visualized IEC systems.

\subsection{Application to Speech Processing}

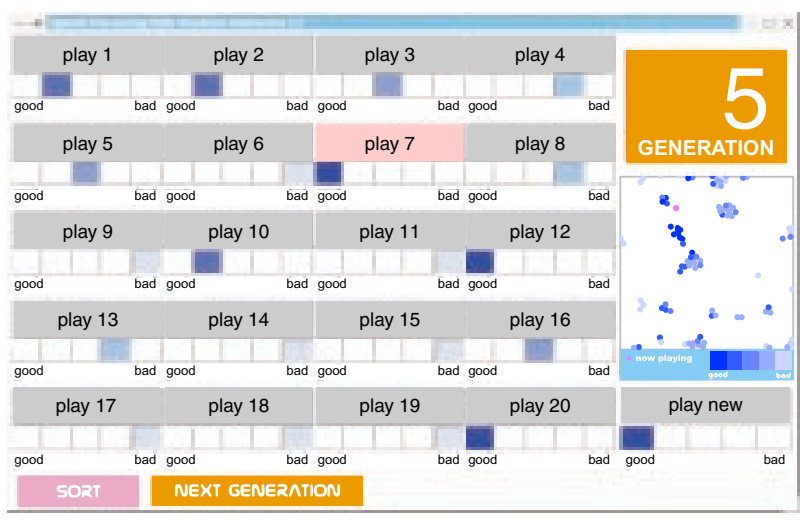

Figure 7: User interface of the Visualized IEC for speech processing.

Figure 7 is a user interface of the Visualized IEC applied to a speech processing system. The IEC speech processing system is a system that improves the quality of speech sound [15]. The frequency characteristics of a filter is specified by 6 parameters of the amplification levels at $125 \mathrm{~Hz}, 250 \mathrm{~Hz}, 500 \mathrm{~Hz}, 1 \mathrm{kHz}, 2 \mathrm{kHz}$, and $4 \mathrm{kHz}$. These parameters are modified to improve the speech quality based on user's hearing and GA search. The processed speech sounds are presented when play buttons on the IEC user interface are pressed. An IEC user evaluates each processed sound, and the GA searches better filter parameters based on user's subjective evaluation as fitness values.

Besides the user interface of the IEC consisting of play buttons and rating buttons for 20 individuals, the interface of the Visualized IEC has a window for data visualization located on the right side of Figure 7. Six dimensional filter parameter vectors are mapped by SOM on the 2-D window and displayed at once. Darker colors are assigned to higher rating buttons, and the same depth of color on the 2-D windows indicates a similar human evaluation of filters in past generations. A Visualized IEC user clicks points where he or she guesses that there are better parameter vectors of filters from the distribution of rated past ones on the 2-D space. Then, a new filter is created from the location information of the clicked point, and the speech sound processed by the filter is displayed to the user. The user evaluates the filter in the same way. The user can repeat to create a better filter until he or she is satisfied, and final created best filter is used as a new parent when the next generation button at the bottom of the figure is pushed.

Figure 7 shows the distribution of the fifth generation of the Visualized IEC for speech processing, and 20 individuals $\times 5$ generations and some individuals newly created by a user are displayed on the 2-D windows. Observing the distribution of fitness values in this figure, it looks like many better individuals are located within the same narrow area on the 2-D window. It seems helpful for the user to quickly find the filter that minimally distorted the speech sound by searching near that place.

\subsection{Application to Room Lighting Design}

We are expanding our previous work of a 3-D CG lighting design support system [1] to a room lighting design. The task of 3-D CG lighting is to determine the best positions and brightness of multiple lights and create a lighting expression by matching the effect to the given motif or personal preference.

Figure 8 is the distribution of 10 room lighting parameter vectors mapped from a $7-\mathrm{D}$ parameter space to a 2-D space. Lighting CG scenes created by 10 lighting parameter vectors are posted in the same figure like as Marks et al.'s Design Galleries [7] for the readers' convenience. Since similar CG scenes tend to gather in the similar location in the 2-D space, it expected that the users could easily and quickly find an ideal lighting for them.

We are going to evaluate the convergence characteristics of the Visualized IEC approach by comparing it with a purely manual searching approach like the Design Galleries [7] or an acoustic data navigator [6].

\section{Conclusion}

We proposed the Visualized IEC that provides the visual landscape of searching space to IEC users and lets them actively participate in EC searching with less fatigue, which results in a faster EC convergence. The 


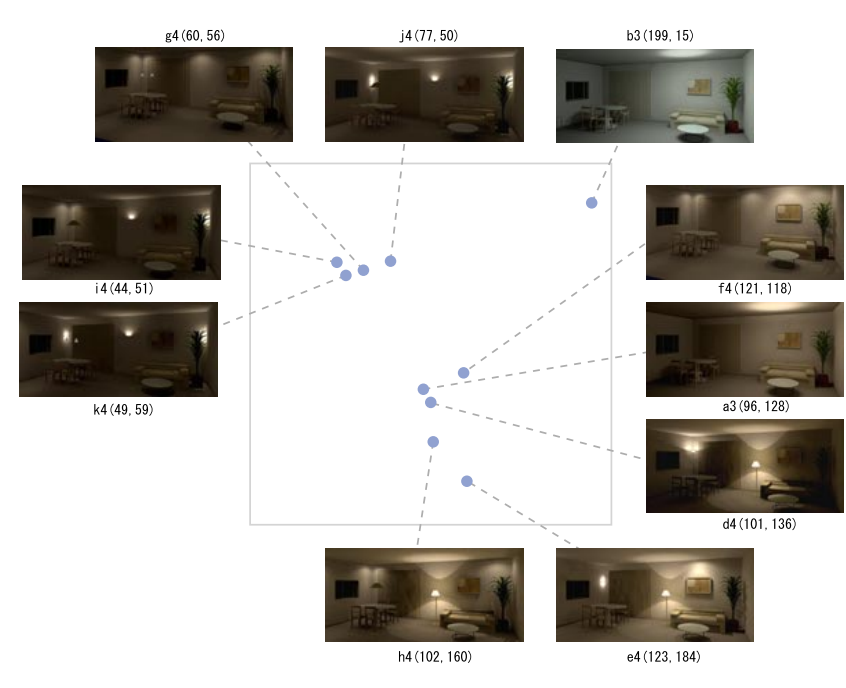

Figure 8: Distribution of room lighting parameters mapped on 2-D space and corresponding CG lighting expression for night scenes.

experimental evaluation implied that the Visualized IEC converges five times faster or more than a normal IEC.

We then showed two example applications of the Visualized IEC, observed the distribution of data in the mapped 2-D space, and found that the individuals whose phenotypes are similar gather and become neighbors in the mapped 2-D space. We are going to continue further evaluation whether a good individual, i.e. a solution of these concrete application tasks, can be found quickly through subjective tests.

Our next step is to compare 2-D visualization methods and find suitable methods for the Visualized IEC to shorten the calculation time, apply the proposed method to several tasks, and evaluate its usefulness.

\section{References}

[1] Aoki, K. and Takagi, H.: "3-D CG lighting with an interactive GA," 1st Int'l Conf. on Conventional and Knowledge-based Intelligent Electronic Systems (KES'97), Adelaide, Australia, pp.296-301 (May, 1997).

[2] Caldwell, C. and Johnston, V. S.: "Tracking a criminal suspect through "face-space" with a genetic algorithm", 4th Int'l Conf. on Genetic Algorithm (ICGA'91), San Diego, CA, US, Morgan Kaufmann Publisher, pp.416421 (July, 1991).

[3] Kohonen, T.: Self-Organizing Maps, Springer-Velag, Heidelberg (1995).

[4] König, A., Bulmahn, O., and Glesner, M.: "Systematic methods for multivariate data visualization and numerical assessment of class separability and overlap in automated visual industrial quality control," 5th British Machine Vision Conf., vol.1, pp.195-204 (Sept., 1994).

[5] König, A.: "A survey of multivariate data projection, visualization and interactive analysis," 5th Int'l Conf. on Soft Computing and Information/Intelligent Systems (IIZUKA'98), Iizuka, Japan: World Scientific, Singapore, pp.55-59 (Oct., 1998).

[6] König, A., Blutner, F. E., Eberhardt, M., and Wenzel, R.: "An acoustic data base navigator for the interactive analysis of psycho-acoustic sound archive," 5th Int'l Conf. on Soft Computing and Information/Intelligent Systems (IIZUKA'98), Iizuka, Japan: World Scientific, Singapore, pp.60-63 (Oct., 1998).

[7] Marks, J., Andalman, B., Beardsley, P. A., et al: "Design Galleries: A general approach to setting parameters for computer graphics and animation," 24th Int'l Conf. on Computer Graphics and Interactive Techniques (SIGGRAPH'97), pp.389-400 (Aug., 1997).

[8] Sammon, J. W.: "A nonlinear mapping for data structure analysis," IEEE Trans. on Computers C-18, no.5, pp.401-409 (1969).

[9] Takagi, H.: "Interactive Evolutionary Computation: System Optimization Based on Human Subjective Evaluation," IEEE Int'l Conf. on Intelligent Engineering Systems (INES'98), Vienna, Austria, pp.1-6 (Sept., 1998).

[10] Takagi, H.: "Interactive Evolutionary Computation - Cooperation of computational intelligence and human KANSEI -," 5th Int'l Conf. on Soft Computing and Information/Intelligent Systems (IIZUKA'98), Iizuka, Fukuoka, Japan: World Scientific, Singapore, pp.41-50 (Oct., 1998).

[11] Takagi, H., Unemi, T., and Terano, T.: "Perspective on interactive evolutionary computing," J. of Japan Society for Artificial Intelligence, vol.13, no.5, pp.692-703 (1998) (in Japanese).

[12] Takagi, H. and Kishi, K.: "On-line knowledge embedding for an interactive EC-based montage system," 3rd Int'l Conf. on Knowledge-Based Intelligent Information Engineering Systems, Adelaide, Australia, pp.280283 (Aug./Sept.,1999).

[13] Venturini, G., Slimane, M., Morin, F., and Asselin de Beauville, J. P.: "On using interactive genetic algorithms for knowledge discovery in databases," 7th Int'l Conf. on Genetic Algorithms, Morgan Kaufmann Publisher, pp.696-703 (1997).

[14] Unemi, T.: "SBART 2.4: breeding 2D CG images and movies and creating a type of collage," 3rd Int'l Conf. on Knowledge-Based Intelligent Information Engineering Systems, Adelaide, Australia, pp.288-291 (Aug./Sept.,1999).

[15] Watanabe, T. and Takagi, H.: "Recovering system of the distorted speech using interactive genetic algorithms," IEEE Int'l Conf. on Systems, Man and Cybernetics, Vancouver, Canada, vol.1, pp.684-689 (Oct., 1995). 